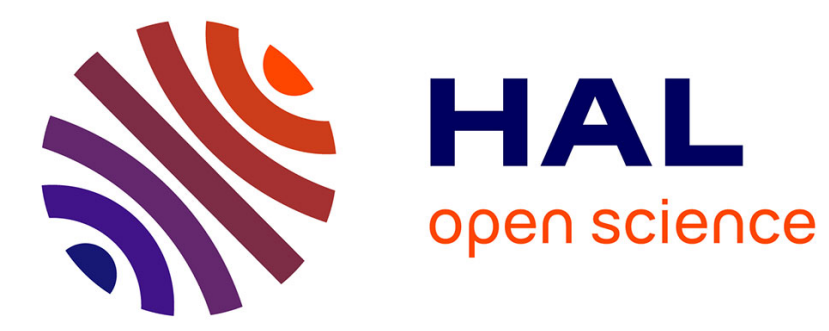

\title{
Progressing Within Amplitude Domain Mechanical Spectroscopy: Overcame Difficulties and Remaining Questions
}

D. Gelli, E. Olzi

\section{To cite this version:}

D. Gelli, E. Olzi. Progressing Within Amplitude Domain Mechanical Spectroscopy: Overcame Difficulties and Remaining Questions. Journal de Physique IV Proceedings, 1996, 06 (C8), pp.C8-281-C8284. 10.1051/jp4:1996860 . jpa-00254668

\section{HAL Id: jpa-00254668 https://hal.science/jpa-00254668}

Submitted on 1 Jan 1996

HAL is a multi-disciplinary open access archive for the deposit and dissemination of scientific research documents, whether they are published or not. The documents may come from teaching and research institutions in France or abroad, or from public or private research centers.
L'archive ouverte pluridisciplinaire HAL, est destinée au dépôt et à la diffusion de documents scientifiques de niveau recherche, publiés ou non, émanant des établissements d'enseignement et de recherche français ou étrangers, des laboratoires publics ou privés. 


\title{
Progressing Within Amplitude Domain Mechanical Spectroscopy: Overcame Difficulties and Remaining Questions
}

D. Gelli and E. Olzi

CNR-ITM, Via Bassini 15, 20133 Milano, Italy

\begin{abstract}
Amplitude domain mechanical spectroscopy is specially interesting as it yields material response data directly in terms of stress or strain amplitudes. Critical stresses and strains are the most immediately significant parameters when material strength is the main object of investigation. On the other hand, as the phenomena are nonlinear, no full rigorous treatise of the complex question of amplitude dependent (material) damping (ADD) and modulus is presently available, as far as we know. However the existence of certain particular, reproducible features of ADD suggest that a systematic analysis of amplitude dependent damping curves (ADD curves) could be carried out by an ad hoc semi-empirical procedure. This procedure, called 'parametric analysis', enables a very precise determination to be given of the various components of ADD. It is thus a means of performing amplitude domain mechanical spectroscopy. An associated model, called 'moties-pinners model' proposes an outline for frequently observed phenomenology in terms of microstructural effects. We shall try here to specify which elements of the treatise can be considered as established and which still require further investigation.
\end{abstract}

\section{INTRODUCTION.}

In all spectroscopic techniques the specimen under test (SUT) is submitted to a given form of incident energy, obviously expressible as a wave, which we call 'the stimulus'. Because of the consequent excitation the SUT emits a given amount of energy, called 'the response'. Spectroscopic analysis is typically carried out by sweeping the frequency of the stimulus and recording the response spectrum (intensity peaks of the remitting energy), versus frequency. This is the frequency domain analysis. The alternative method, which is to sweep the amplitude of the stimulus instead of its frequency, is the amplitude domain analysis. This latter alternative has been very seldom - almost never - exploited in conventional spectroscopy, a fact which is probably due to a generalised reluctance to address non-linear phenomena.

Mechanical spectroscopy is also mainly carried out in the frequency domain, sometimes by a true frequency sweep or, most often, by a ruse based on continuous temperature variation. However interest in the amplitude domain analysis of mechanical spectroscopy has not been so rare as it has in the other spectroscopic techniques. Proof of this is the attention which has been given to amplitude dependent damping (ADD) by materials scientists. In fact ADD (and modulus) curves contain information regarding amplitude domain. analysis, but only in an implicit form. It is, however, possible to render such information more explicit by 'parametric analysis' [1-3], which enables us to separate the various components of the ADD curves, thus rendering the data much more consistent with what, in the language of spectroscopy, is meant by a 'spectrum'..

A very synthetic overview is given here, citing a practical example of what we mean by 'amplitude domain spectrum', the semi-quantitative model we are using to interpret it and the aspects which, in our opinion, have been cleared up and those which still remain on the table.

\section{AN EXAMPLE OF AMPLITUDE DOMAIN SPECTRUM.}

We have used for this example an ADD curve by Jon, Mason and Beshers on OFHC polycrystalannealed-copper [4, fig. 2]. The curve considered was obtained at $22.5 \mathrm{kHz}$ by longitudinal excitation of the sample, at room temperature and it expresses the variation of internal friction $\mathrm{Q}^{-1}$ with strain amplitude $\varepsilon$. They are measurements made point by point in stationary conditions. The authors did not observe 'time effects'. However parametric analysis is also applicable where these are present. 

obtained.

When the curve is analysed parametrically (1st stage, see later on) the diagram shown in Fig. 1a) is

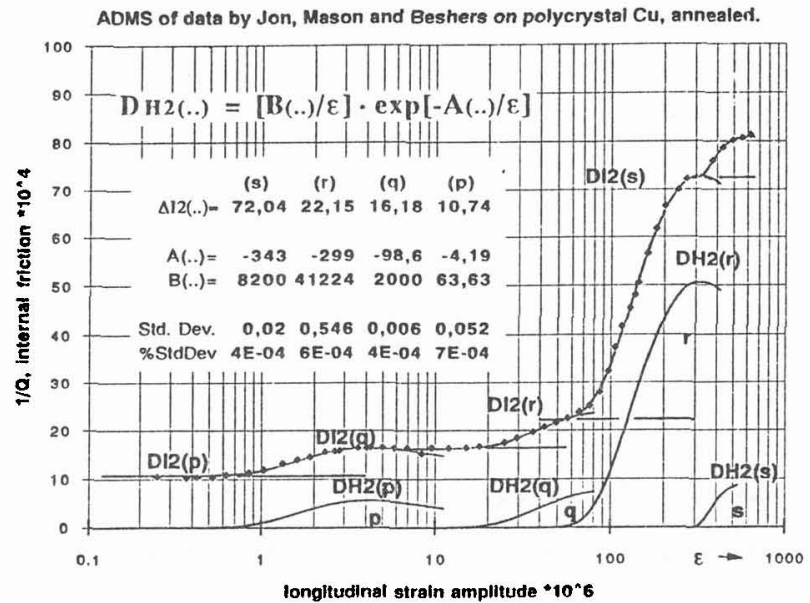

a)

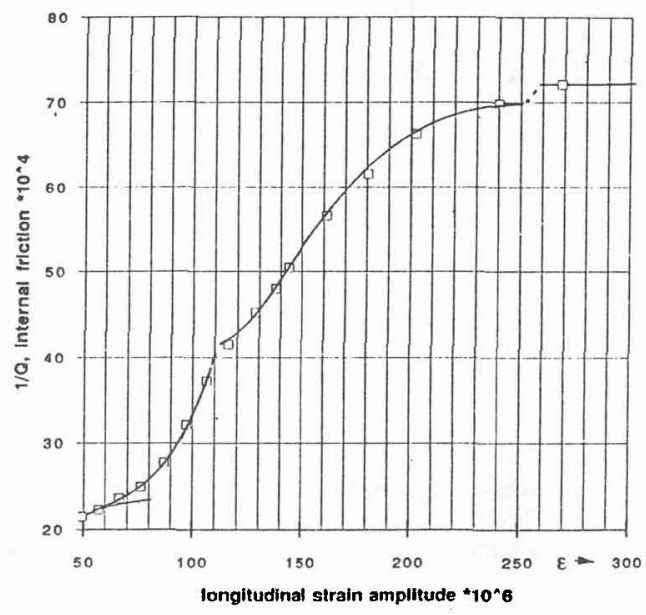

b)

Figure 1: a) Example of an Amplitude Domain Spectrum (ADS) as obtained by parametric analysis of amplitude dependent internal friction data.

b) Magnified view of the preceding graph, in the ' $r$ ' amplitude range: a parabolic-sigmoidal hypothesis gives a better fitting than an all sigmoidal ('all GL') hypothesis.

As can be seen the graph can be separated into four strain-amplitude ranges indicated as: $\mathrm{p}, \mathrm{q}, \mathrm{r}$, and $\mathrm{s}$. In each of these the internal friction can be represented as the sum of an amplitude independent damping component

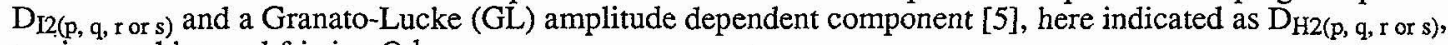
to give total internal friction $\mathrm{Q}^{-1}$.

The table superimposed on the graph shows, for each sub-range $\mathrm{p}, \mathrm{q}, \mathrm{r}$ and $\mathrm{s}$, the value of the relevant

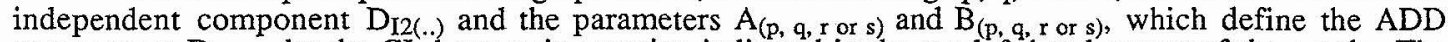
components $\mathrm{D}_{\mathrm{H} 2(. .)}$ by the GL hysteresis equation indicated in the top left-hand corner of the graph. The $D_{\mathrm{H} 2(. .)}$ dependent type components appear as something resembling peaks in the lower part of the graph. The - $\mathrm{D}_{\mathrm{I} 2(. .)}$ independent type components appear above them as a steplike sequence of horizontal lines. They are, together with the parameters $\mathrm{A}_{(. .)}$and $\mathrm{B}_{(. .)}$, the result of an iteration process.

The interpolation curve, which is obtained by adding the above components together, appears further up on the graph, close to the experimental points. And can be seen to be a good interpolating curve. It is also possible to examine the interpolation fit further by using the standard deviation data (total or percent), also included in the table in Fig. 1a. These data express the extent to which the experimental points fit well onto each relevant 'theoretical' GL curve. It may be seen that the fitting is very good or good for the $p, q$ and $s$ components, while for the $\mathrm{r}$ component it is rather less good.. To find the reason for this, the part of the graph regarding the $\mathrm{r}$ component is magnified in Fig. 1b. Here it can be seen that although the fitting would not be very bad with the 'all GL hypothesis' (first stage of the Analysis) it can be appreciably improved with a LinearParabolic-Sigmoidal (LPS) hypothesis. Looking for LPS segments of the ADD curve is the Analysis' 2nd stage (and refinement).

The possible coexistence of one or more sequences of LPS segments in an ADD curve is another established fact [6]. We call the rectilinear trend the Takahashi trend, the parabolic trend the Read-Nowick trend and the sigmoidal trend the GL trend. The possibility to split the $\mathrm{r}$ peak into a Takahashi, a Read-Nowick and a GL component can be seen as the existence of 'a fine structure' in the $r$ peak.

The diagram in Fig. 1a splits the original ADD curve into its peaks as a function of amplitude. It therefore appears as the dual of the frequency domain spectrum when amplitude substitutes frequency. This is why we call such a diagram 'Amplitude Domain Spectrum' (ADS). An ADS so obtained is an easily readable identity card of the technological and structural state of the SUT. Thanks to the peculiar sensitivity of internal friction to small microstruetural variations, this spectrum will also be an extremely sensitive monitor of the effects of very small thermal, plastic or thermo-mechanical treatments and the parameters $A_{(. .)}, B_{(. .)}$(and then a, $\alpha, \beta$ and the critical amplitudes $\varepsilon_{\mathrm{j}}$, see refs. 1-3) are numbers that give in a more or less empirical way a measure of the variations induced by the treatments. 
Now, by leaving out many other considerations, we can conclude that parametric analysis is by itself a way to perform Amplitude Domain Mechanical Spectroscopy. And this is already an appreciable result.

But to make ADMS the powerful investigation tool required by material science and engineering, a model is needed for a physical interpretation of the observed phenomenology, in terms of the microstructure of the material under test. We are now working on a model we have called the 'moties-pinners (MP) model' [6] which, in our opinion, is highly congruous with the recurring phenomenology expressed by ADD curves, and even more so by ADSs of the type exemplified in Figs. 1a (and b). In pursuing this model we are following the guidelines set out below.

\section{LINES FOR A GENERAL MODEL: THE MOTIES-PINNERS MODEL}

The guidelines are based on the fact that a material matrix contains:

i) a number of structural entities, mobile entities, or 'moties', liable to move under the effect of internal stresses, and

ii) another kind of structural entity, the possible pinning agents, or 'pinners', which can be an obstacle to moty movement.

Some examples of moties are; single dislocations, dislocation structures, stacking faults, subgrain boundaries, grain boundaries, twin boundaries and interphase boundaries, magnetic domain boundaries, magnetic flux lines, polymeric chains, etc. (point defects are not considered).

Some examples of pinners are; solute atoms, vacancies, jogs, dislocation intercepts, solute atom aggregates like Guinier Preston zones, heterogeneous components like reprecipitates or dispersed phases, interceptions between embedded fibres or polymeric chains.

Entities which are not just line defects have been included with the possible moties. Similarly, entities which look much more complex than point defects have been included with the possible pinners. In this 'moties-pinners model', the hysteretic contribution to material damping, connected with breakaway phenomena (of moties from pinners), is expected to play a role and a similarity is expected with what is provided by the GL model, where dislocations only are involved.

We have therefore advanced a new hypothesis and at the same time introduced a new concept [7]. The new hypothesis is that any moty can be modelled by an equivalent dislocation or dislocation similarity or quasidislocation or, more simply, by an equivalent pinned string. The new concept is the concept of the 'equivalent dislocation of a given moty'. While this hypothesis might seem almost spontaneous for moties of linesymmetry like dislocations, it appears somewhat less so in the case of defects such as subgrain-boundaries, grain-boundaries, magnetic domain boundaries, etc. which are not linear but area-like defects. Despite this, it is our opinion that it should not be excluded that the movement of a pinned two-dimensional defect can also be reduced to the movement of an equivalent pinned string. This is an agreement with the concept of 'scaling' that has been expressed e.g. by Hilzinger [8]. We have reached this conviction because most experimental ADD results concerning the two-dimensional defects (specially domain and interphase boundaries) show behaviour which is fully assimilable with the string-like behaviour of pinned dislocations [7].

\section{DISCUSSION AND CONCLUSION}

According to this MP model, the various pairs of 'steps' and 'peaks' such as $\mathrm{D}_{\mathrm{I} 2(. .)}$ and $\mathrm{D}_{\mathrm{H} 2(. .)}$, in the ADS of Fig. Ia, are dynamic and hysteretic contributions due to the alternating movement of moties which, while still attached to certain pinners at the lower limit of a given subrange (p, $q, \mathrm{r}$ or $\mathrm{s}$ ), gradually break away, finally leaving them totally when the amplitude $\varepsilon$ reaches the subrange's upper limit.

The identity of the moty-pinner pairs operating critically in the various subranges is not necessarily known a priori. If necessary it may be identified by complementary investigation or other evaluations.

What follows can be commented in some greater detail.

a) The fact that one or more GL segments can be seen in a same ADD curve could be ascribed to one or more moty-pinner systems being able to become critical in turn, at the various incremental amplitudes. In the example of Fig. 1a, the sigmoidal components $D_{\mathrm{H} 2(\mathrm{p})}$ and $\mathrm{D}_{\mathrm{H} 2(\mathrm{q})}$ would represent two subsequent breakaway events relevant to two different MP systems operating in the amplitude ranges $p$ and $q$. In cases like these, direct information can be drawn by the GL equations if dislocations are mainly concerned. Some indirect information could be drawn via the equivalent dislocation principle, when other moties are involved. Indeed quantitative data can be obtained from the sigmoidal (GL) segments (e.g. via GL plotting), concerning dislocation (moties) density, interpinner distance etc.

b) It is not so easy to obtain quantitative microstructural information from the linear and parabolic segments of the curves, if there are any. Despite the large amount of literature on ADD curves with linear and parabolic trends, we know of no compliant and exhaustive treatise of the phenomena. A dislocation-damping treatise involving linear and parabolic components has been advanced by Friedel [9], but it has been employed rarely. Why this should be is not clear except perhaps for the eventual difficulty of the algorithms involved. 
c) A possible repetition of the LPS sequence in a same ADD curve should not cause surprise, given the possible intervention of different moty-pinner systems in different amplitude ranges, each system being able to generate an LPS sequence.

d) The existence of one or more maxima in an ADD curve should no longer be considered anomalous, given the large number of experimental curves available involving maxima. Examples pertaining to different materials and measuring conditions have already been reported on a previous occasion [7]. Neither should these maxima cause surprise. The GL 'breakaway law' itself [5] intrinsically involves a maximum. In the GL picture the observed maxima could simply be interpreted as the conclusions of dislocation-breakaway phenomena. In the moty-pinner picture, and taking into account the 'equivalent dislocation principle' again, each maximum could represent the breakaway of a class of moties from their last critical class of pinners.

e) The plurality of amplitude independent components such as $\mathrm{D}_{\mathrm{I} 2(. .)}$ in Figure 1a could be regarded as the (reasonably dynamic) contribution of all the moties already set free due to the breakaway events that have occurred so far. In the MP view all this behaviour would seem to indicate that each time a moty undergoes a breakaway or simply changes its way of moving inside its pinner cloud [2], a new dynamic component arises.

Another fact must also be recorded. In most instances, especially in the kilohertz range, any newly emerging amplitude independent damping component nearly equals the last attained (maximum) value of the total damping.

No theories are presently available to describe these a.i. components quantitatively. And this is one of the unsolved 'remaining questions'.

\section{ACKNOWLEDGEMENTS.}

Thanks are due to Pr. D. N. Beshers for useful discussions and Mrs. R.V. Hunt for revising the English.

\section{REFERENCES}

[1] Gelli, D., Physica Status Solidi , 12, 1965, 829-841 and K 109-K112.

[2] Gelli, D., Alluminio Nuova Metallurgia, 36, 1967, 511 - 519.

[3] Gelli, D., Journal de Physique, Supplement au n 7, 32, 1971, C2 137 - C2 143.

[4] Jon, M.C.,Mason, W.P. and Beshers, D.N., Journ. Appl. Phys., 47, 1976, 2337 - 2349.

[5] Granato, A., Lücke, K., Journ. Appl. Phys. , 27,1956, 789-805.

[6] Gelli, D., Olzi, E., Journal of Alloys Compounds , 211/212 , 1994, pp. 603-607.

[7] Gelli, D., Olzi, E., "Amplitude Dependent Material Damping: Old Beliefs and New Facts. Working to a Synthesising Model", International Symposium On M ${ }^{3} \mathrm{D}$ III Mechanics and Mechanism of Material Damping, Norfolk, Nov. 12-17, 1995, ASTM-STP 1304 (in presse).

[8] Hilzinger, H. R., Philosophical Magazine, 36, 1977, 225-234.

[9] Friedel, J., " On Amplitude Dependent Internal Friction" The Relation Between the Structure and Mechanical Properties of Metals, Allen N.P., Ed. (Her Majesty's Stationery Office, London, 1963) 410 $-427$. 